\title{
Doping-induced disappearance of ice II from water's phase diagram
}

\author{
Jacob J. Shephard, Ben Slater, Peter Harvey, \\ Martin Hart, Craig L. Bull, Steven T. Bramwell \\ and Christoph G. Salzmann
}

\section{Published version information}

Citation: JJ Shephard et al. "Doping-induced disappearance of ice II from water's phase diagram." Nature Physics, vol. 14 (2018) 569-572.

DOI: $\underline{10.1038 / s 41567-018-0094-z}$

This version is made available in accordance with publisher policies. Please cite only the published version using the reference above. This is the citation assigned by the publisher at the time of issuing the AAM/APV. Please check the publisher's website for any updates. 


\section{Doping-induced Disappearance of Ice II from Water's}

\section{Phase Diagram}

Jacob J. Shephard, ${ }^{a}$ Ben Slater, ${ }^{a}$ Peter Harvey, ${ }^{a}$ Martin Hart, ${ }^{a}$ Craig L. Bull, ${ }^{\text {b }}$ Steven T. Bramwell ${ }^{\mathrm{c}}$ and Christoph G. Salzmann*a

${ }^{a}$ Department of Chemistry, University College London, 20 Gordon Street, London WC1H OAJ, UK.

${ }^{b}$ ISIS Facility, Rutherford Appleton Laboratory, Didcot, OX11 OQX, UK.

${ }^{c}$ London Centre for Nanotechnology and Department of Physics \& Astronomy, University College London, 17-19 Gordon Street, London WC1H OAJ, U.K.

email: c.salzmann@ucl.ac.uk

tel.: +44 (0) 2076798864 
Water and the many phases of ice display a plethora of complex physical properties and phase relationships ${ }^{1-4}$ which are of paramount importance in a range of settings including processes in Earth's hydrosphere, the geology of icy moons, industry and even the evolution of life. Well-known examples include the unusual behaviour of supercooled water, ${ }^{2}$ the emergent ferroelectric ordering in ice films ${ }^{4}$ and the fact that the 'ordinary' ice I $h$ floats on water. We report the intriguing observation that ice II, one of the high-pressure phases of ice, disappears in a selective fashion from water's phase diagram upon addition of small amounts of ammonium fluoride. This finding exposes the strict topologically-constrained nature of the ice II hydrogen-bond network which is not found for the competing phases. In analogy to the behaviour of frustrated magnets, ${ }^{5}$ the presence of the exceptional ice II is argued to have a wider impact on water's phase diagram potentially explaining its general tendency to anomalous behaviour. Furthermore, the impurity-induced disappearance of ice II raises the prospect that specific dopants may not only be able to suppress certain phases but also induce the formation of new phases of ice in future studies.

Whenever water freezes at a given pressure, hydrogen-disordered phases of ice crystallize which display orientational disorder of the hydrogen-bonded water molecules. ${ }^{3,6,7}$ The corresponding hydrogen-ordered counterparts exhibit long-range orientational order and form upon cooling as required by the $3^{\text {rd }}$ law of thermodynamics. ${ }^{8-10}$ The one notable exception, that displays a fundamentally different behaviour compared to the other ice phases, is ice II whose region of stability dominates the phase diagram in the pressure range up to $1 \mathrm{GPa}$ (Figure 1a). Ice II remains hydrogen-ordered up to temperatures $6^{\circ} \mathrm{C}$ below the melting point, ${ }^{11,12}$ whereupon it undergoes phase transitions to the hydrogen-disordered ices I $h$, III, V or VI rather than to its own hydrogendisordered counterpart. Recent computational studies have shown that the phase transition from ice II to the hypothetical hydrogen-disordered ice II $d$ would only take place at temperatures well within 
the stability region of the liquid highlighting a strong resilience of ice II towards hydrogen disordering. ${ }^{13,14}$

Here we test the response of water's phase diagram with respect to a specific disturbance, the addition of small amounts of ammonium fluoride $\left(\mathrm{NH}_{4} \mathrm{~F}\right)$. While $\mathrm{NH}_{4} \mathrm{~F}$ is well-known to mix with the 'ordinary' ice I $h$ across a large composition range ${ }^{15,16}$ and has been shown to incorporate into clathrate hydrates, ${ }^{17,18}$ the mixing of $\mathrm{NH}_{4} \mathrm{~F}$ with high-pressure phases of ice has not been previously investigated. The local hydrogen-bonding environment of ice is in principle well-adapted for incorporating $\mathrm{NH}_{4}{ }^{+}$and $\mathrm{F}^{-}$ions in terms of the length, strength and number of hydrogen bonds. ${ }^{19}$ Yet, a major chemical difference between $\mathrm{H}_{2} \mathrm{O}, \mathrm{NH}_{4}{ }^{+}$and $\mathrm{F}^{-}$lies in the different numbers of hydrogen bonds they can donate or accept. Consequently, as shown schematically in Figure 1b, the replacement of a $\mathrm{H}_{2} \mathrm{O}$ molecule in ice with $\mathrm{NH}_{4}{ }^{+}$or $\mathrm{F}^{-}$requires the reorientations of two neighbouring water molecules.

Remarkably, the presence of small amounts of $\mathrm{NH}_{4} \mathrm{~F}$ leads to the disappearance of the hydrogen-ordered ice II from water's phase diagram and the hydrogen-disordered ices III and V are found instead (Figure 1c). Ice II appears to be incompatible with $\mathrm{NH}_{4} \mathrm{~F}$ whereas the other phases of ice can incorporate the impurity which leads to a shift of the onsets of melting towards lower temperatures. Unlike for pure ice, the melting of the two-component solid solutions is expected to take place over a temperature range. Figure 2 a shows neutron diffraction data of $\mathrm{D}_{2} \mathrm{O}$ ice with 2.5 mol\% $\mathrm{ND}_{4} \mathrm{~F}$ collected at $0.3 \mathrm{GPa}$ with argon gas as the pressure medium. The doped ice III, which exists instead of ice II in the phase diagram of pure ice, is present upon heating from $200 \mathrm{~K}$ to its final melting point at $\sim 255 \mathrm{~K}$. Due to the high argon pressure, argon clathrate hydrate forms in the temperature range of melting.

Upon lowering the mole fraction of $\mathrm{NH}_{4} \mathrm{~F}$, the ice III persists down to $0.5 \mathrm{~mol} \%$ at $0.3 \mathrm{GPa}$. Figure $1 \mathrm{~d}$ shows the volume changes of metastable ice $\mathrm{I} h / \mathrm{NH}_{4} \mathrm{~F}$ solid solutions recorded upon isobaric heating at $0.3 \mathrm{GPa}$. Below $0.5 \mathrm{~mol} \%$, the ice III 'plateau' drops towards the 'basin' of denser ice II which must form upon approaching pure ice. The appearance of ice II at $0.25 \mathrm{~mol} \%$ 
$\mathrm{ND}_{4} \mathrm{~F}$ was investigated with neutron diffraction at $0.3 \mathrm{GPa}$. Figure $2 \mathrm{~b}$ shows that a metastable stage of ice III is first observed at $200 \mathrm{~K}$. Upon heating to $235 \mathrm{~K}$, the ice III transforms to $\sim 80 \mathrm{w} \%$ ice II while $\sim 20 \mathrm{w} \%$ of ice III remain. The metastable nature of the initial ice III is demonstrated by cooling back to $180 \mathrm{~K}$ during which the weight fraction of ice II increases gradually. As observed for pure ice II, it persists up to $245 \mathrm{~K}$ upon subsequent heating where transformation to ice III and finally melting is observed.

Ice II appears at nominally $0.25 \mathrm{~mol} \% \mathrm{ND}_{4} \mathrm{~F}$, yet the actual solubility of $\mathrm{ND}_{4} \mathrm{~F}$ in ice II is much smaller. As it has been shown for solid solutions of ice $\mathrm{I} h$ and $\mathrm{NH}_{4} \mathrm{~F}$, the lattice constants are sensitive indicators for the amount of $\mathrm{NH}_{4} \mathrm{~F}$ mixed into the ice which leads to subtle contractions of the lattice constants. ${ }^{19}$ The $a$ lattice parameter of $\mathrm{D}_{2} \mathrm{O}$ ice III with $2.5 \mathrm{~mol} \% \mathrm{ND}_{4} \mathrm{~F}$ determined from the neutron diffraction data is $6.66015 \pm 0.00007 \AA$ at $0.3 \mathrm{GPa}$ and $200 \mathrm{~K}$. The corresponding value for the metastable ice III with $0.25 \mathrm{~mol} \% \mathrm{ND}_{4} \mathrm{~F}$ is $0.100 \pm 0.001 \%$ greater at the same pressure and temperature. After transforming the $0.25 \mathrm{~mol} \% \mathrm{ND}_{4} \mathrm{~F}$ sample to ice II upon heating and cooling back to $200 \mathrm{~K}$, the $a$ lattice parameter of the $\sim 10 \mathrm{w} \%$ ice III is within error the same as observed for the $2.5 \mathrm{~mol} \%$ sample. This means that the vast majority of $\mathrm{ND}_{4} \mathrm{~F}$ is located within the ice III and that the solubility of $\mathrm{ND}_{4} \mathrm{~F}$ in ice II is very small. This is also illustrated by the fact that the neutron diffraction data of the ice II in the $0.25 \mathrm{~mol} \%$ sample is consistent with pure and fully hydrogenordered ice II (see Supplementary Information Section 2).

The exclusion of $\mathrm{ND}_{4} \mathrm{~F}$ from the growing ice II crystals indicates that this process is governed by the underlying thermodynamics and that a high free-energy cost is associated with the incorporation of $\mathrm{ND}_{4} \mathrm{~F}$ into ice II. Density-functional-theory calculations show that the local substitution energies of $\mathrm{NH}_{4} \mathrm{~F}$ into ices II $d$ and III are very similar (see Supplementary Information Section 3). Consequently, the different responses of ices II and III to $\mathrm{NH}_{4} \mathrm{~F}$ must be due to differences in the characteristics of their hydrogen-bonded networks.

The inability of the hydrogen-ordered ice II to incorporate ammonium fluoride suggests that its configurational manifold is "topologically constrained". This means that the ice rules in ice II are 
strictly enforced, and that thermally-excited ionic $\left(\mathrm{H}_{3} \mathrm{O}^{+}\right.$and $\left.\mathrm{OH}^{-}\right)$and Bjerrum defects $(\mathrm{O} \cdots \mathrm{O}$ and $\mathrm{O}-\mathrm{H} \cdots \mathrm{H}-\mathrm{O}$ ) are completely absent which is consistent with dielectric spectroscopy measurements. ${ }^{20}$ Its topologically-constrained nature therefore clearly distinguishes ice II from the competing phases in the phase diagram. Similar to topologically-constrained manifolds in ferroelectrics, ${ }^{21}$ spin ice ${ }^{22,23}$ and biomembranes,${ }^{24}$ the ice-rule manifold of ice II exhibits the remarkable property of zero entropy and a fully-ordered hydrogen structure at relatively high temperatures, ${ }^{12}$ despite the availability of a vast number of hydrogen-disordered states.

Figure 3a shows schematically the problem associated with the incorporation of $\mathrm{F}^{-}$and $\mathrm{NH}_{4}{ }^{+}$ ions at random into the topologically-constrained ice II manifold. With no ionic and Bjerrum defects available to screen them, the dopants enter the constrained manifold of ice-rule states as topological defects. ${ }^{25}$ This can be rationalized by constructing a flux of hydrogen displacement vectors in a manner analogous to spin-ice mapping ${ }^{26}$ and is most easily visualized for the twodimensional square ice and if the orientational order is considered to be ferroelectric (Figure 3a). The flux lines of hydrogen displacement are then parallel in the hydrogen-ordered state. Upon $\mathrm{NH}_{4} \mathrm{~F}$ doping, the flux lines must connect two dopant sites which are expected to be strongly entangled and interwoven in three-dimensional ice structures. The dopants therefore act as topological defects in the hydrogen-displacement flux, charge-like sources and sinks of flux ${ }^{27}$ that disrupt the orientational order of the water molecules over long distances along a system of flux tubes between the $\mathrm{NH}_{4}{ }^{+}$and $\mathrm{F}^{-}$defects. The enthalpy cost per ion then scales at least as $\sim x^{-1 / 3}$, where $x$ is the dopant fraction, and low-level doping comes at a high free-energy cost per ion. This does not apply to the competing ices I $h$, III, V and VI because of the absence of orientational order and the availability of Bjerrum defects to screen the topological charges.

The formation of closely bound $\mathrm{NH}_{4}{ }^{+}-\mathrm{F}^{-}$pairs would of course reduce the energy of the randomly-doped system illustrated in Figure 3a, but it turns out that on the topologicallyconstrained ice rules manifold of ice II, this cannot eliminate flux tubes of significant length. In either scenario, the overall result is a significant increase in free energy per dopant ion, sufficient to 
preclude the absorption of $\mathrm{NH}_{4} \mathrm{~F}$ into the ice II structure. Full details of the statistical mechanics and the topology of the ice II network as well as experimental arguments against defect pairing are presented in the Supplementary Information Section 4.

The corresponding thermodynamics are illustrated in Figure $3 \mathrm{~b}$ which shows schematic free energy versus temperature curves. The stable phase is the one with the lowest Gibbs free energy and the slopes of the curves reflect the entropy, $S$, according to $(\partial G / \partial T)_{p}=-S$. Ice II suffers a substantial free energy increase in the presence of $\mathrm{NH}_{4} \mathrm{~F}$ arising from the long-range disruption of its periodic order. This raises its free energy towards the hydrogen-disordered ice II $d$ which is metastable at all temperatures and therefore absent from the phase diagram.

The topologically-constrained nature of ice II clearly distinguishes it from the neighbouring phases in the phase diagram and we argue that the ice II anomaly has a wider impact on the water phase diagram. Generally speaking, the higher the entropy of a solid the greater its thermal stability with respect to competing phases (through $G=H-T S$ ). Although ice II is energetically very stable, its lack of configurational entropy means that it eventually becomes thermally unstable above a certain temperature with respect to more disordered phases (Figure 3b). Assigning ice II an interaction-energy scale of $-k \theta$ per water molecule relative to its competing phases means that the temperature range of existence of the competing phases, the hydrogen-disordered ices I $h, \mathrm{III}, \mathrm{V}$ and VI as well as the liquid, is anomalously broadened towards low temperature, $T<\theta$. As in the case of frustrated magnets such pyrochlore spin ices or kagome spin liquids, ${ }^{5}$ the persistence of disordered states down to temperatures lower than the mean-field ordering temperature $T_{\mathrm{o}} \sim \theta$ implies that the disordered degrees of freedom in these phases are much more strongly correlated than they would be if they only occurred above $T_{\mathrm{o}}$. In a sense, the disordered phases are energetically drawn towards the topologically-constrained ice II state. Yet, the ice II order is precluded by its cost in entropy, at least on extended length scales. The consequence is a host of anomalous properties and phase transitions in the disordered solid and liquid states, as has been widely documented. ${ }^{1-4}$ The constrained topology of ice II therefore not only determines its own 
special character but it may also provide a deeply-rooted explanation for the existence of a host of anomalous condensed states that compete for stability in water's phase diagram.

The doping-induced disappearance of ice II also provides a remarkable example for the impact an impurity can have on the appearance of the phase diagram of ice. Knowledge of such effects is obviously important whenever ice coexists with other materials in nature. But it also raises the exciting prospect of instead of removing phases from the phase diagram to potentially favouring the formation of new phases of ice in the presence of specific dopants. Ammonium-fluoride doping is expected to induce hydrogen disorder in all the hydrogen-ordered phases of ice. The fully hydrogen-ordered phases should be most responsive due to their inabilities in screening the topological charges. In addition to ice II, we therefore predict the fully hydrogen-ordered ice VIII ${ }^{28}$ to undergo substantial free-energy increases in the presence of small amounts of ammonium fluoride. This makes its large region of stability above $\sim 2$ GPa particularly promising for finding new phases in future studies.

\section{Methods}

Methods, including statements of data availability and any associated accession codes and references, are available in the online version of this paper.

\section{References}

1 Canton, J. Experiments and Observations on the Compressibility of Water and Some Other Fluids. J. Philosophical Transactions 54, 261-262, (1764).

2 Gallo, P. et al. Water: A Tale of Two Liquids. Chem. Rev. 116, 7463-7500, (2016).

3 Salzmann, C. G., Radaelli, P. G., Slater, B. \& Finney, J. L. The Polymorphism of Ice: Five Unresolved Questions. Phys. Chem. Chem. Phys. 13, 18468-18480, (2011). 
4 Sugimoto, T., Aiga, N., Otsuki, Y., Watanabe, K. \& Matsumoto, Y. Emergent high-Tc ferroelectric ordering of strongly correlated and frustrated protons in a heteroepitaxial ice film. Nat. Phys. 12, 1063-1068, (2016).

5 Ramirez, A. P. Strongly Geometrically Frustrated Magnets. Annu. Rev. Mater. Sci. 24, 453480, (1994).

6 Petrenko, V. F. \& Whitworth, R. W. Physics of Ice. (Oxford University Press, 1999).

7 Salzmann, C. G., Radaelli, P. G., Hallbrucker, A., Mayer, E. \& Finney, J. L. The Preparation and Structures of Hydrogen Ordered Phases of Ice. Science 311, 1758-1761, (2006).

8 Pauling, L. The structure and entropy of ice and other crystals with some randomness of atomic arrangement. J. Am. Chem. Soc. 57, 2680-2684, (1935).

9 Tajima, Y., Matsuo, T. \& Suga, H. Phase Transition in KOH-doped Hexagonal Ice. Nature 299, 810-812, (1982).

10 Salzmann, C. G., Radaelli, P. G., Mayer, E. \& Finney, J. L. Ice XV: A New Thermodynamically Stable Phase of Ice. Phys. Rev. Lett. 103, 105701, (2009).

11 Lobban, C., Finney, J. L. \& Kuhs, W. F. The p-T dependency of the ice II crystal structure and the effect of helium inclusion. J. Chem. Phys. 117, 3928-3834, (2002).

12 Whalley, E. \& Davidson, D. W. Entropy changes at the phase transitions in ice. J. Chem. Phys. 43, 2148-2149, (1965).

13 Fan, X., Bing, D., Zhang, J., Shen, Z. \& Kuo, J.-K. Predicting the hydrogen bond ordered structures of ice Ih, II, III, VI and ice VII: DFT methods with localized based set. Comput. Mater. Sci. 49, S170-S175, (2010).

14 Nakamura, T., Matsumoto, M., Yagasaki, T. \& Tanaka, H. Thermodynamic Stability of Ice II and Its Hydrogen-Disordered Counterpart: Role of Zero-Point Energy. J. Phys. Chem. B 120, 1843-1848, (2016).

15 Brill, R. \& Zaromb, S. Mixed Crystals of Ice and Ammonium Fluoride. Nature 173, 316-317, (1954). 
16 Labowitz, L. C. \& Westrum, E. F. A Thermodynamic Study of the System Ammonium Fluoride-Water. II. The Solid Solution of Ammonium Fluoride in Ice. J. Phys. Chem. 65, 408414, (1961).

17 Shin, K. et al. Crystal engineering the clathrate hydrate lattice with $\mathrm{NH}_{4} \mathrm{~F}$. CrystEngComm 16, 7209-7217, (2014).

18 Park, S., Lim, D., Seo, Y. \& Lee, H. Incorporation of ammonium fluoride into clathrate hydrate lattices and its significance in inhibiting hydrate formation. Chem. Comm. 51, 87618764, (2015).

19 Lyashchenko, A. K. \& Malenkov, G. G. X-ray Investigation of Ammonium Fluoride - Ice Systems. Zhurnal Strukturnoi Khimii 10, 724-725, (1969).

20 Wilson, G. J., Chan, R. K., Davidson, D. W. \& Whalley, E. Dielectric properties of ices II, III, V, and VI. J. Chem. Phys. 43, 2384-2391, (1965).

21 Slater, J. C. Theory of the Transition in $\mathrm{KH}_{2} \mathrm{PO}_{4}$. J. Chem. Phys. 9, 16-33, (1941).

22 Jaubert, L. D. C., Chalker, J. T., Holdsworth, P. C. W. \& Moessner, R. Spin Ice under Pressure: Symmetry Enhancement and Infinite Order Multicriticality. Phys. Rev. Lett. 105, 087201, (2010).

23 Powell, S. Higgs transitions of spin ice. Phys. Rev. B 84, 094437, (2011).

24 Nagle, J. F. Theory of biomembrane phase transitions. J. Chem. Phys. 58, 252-264, (1973).

25 Onsager, L. \& Dupuis, M. in Thermodinamica dei processi irreversibili, Rendiconti della Scuola Internazionale di Fisica "E.Fermi", Corso 10 294-315 (1960).

26 Bramwell, S. T. \& Harris, M. J. Frustration in Ising-type spin models on the pyrochlore lattice. J. Phys. Condens. Matter 10, L215, (1998).

27 Ryzhkin, I. A. Magnetic relaxation in rare-earth oxide pyrochlores. J. Exp. Theor. Phys. 101, 481-486, (2005).

28 Kuhs, W. F., Finney, J. L., Vettier, C. \& Bliss, D. V. Structure and hydrogen ordering in ices VI, VII, and VIII by neutron powder diffraction. J. Chem. Phys. 81, 3612-3623, (1984). 
29 Kamb, B., Hamilton, W. C., La Placa, S. J. \& Prakash, A. Ordered Proton Configuration in Ice II, from Single-Crystal Neutron Diffraction. J. Chem. Phys. 55, 1934-1945, (1971).

\section{Acknowledgements}

We thank the Royal Society (UF150665) and the Leverhulme Trust (RPG-2014-04) for funding, the ISIS facility for granting access to the PEARL instrument, Chris Ridley for help with the PEARL pressure equipment, M. Vickers for help with the X-ray measurements, J. K. Cockcroft for access to the Cryojet, and S. L. Price, A. K. Soper and P. A. McClarty for helpful discussions. We also acknowledge the use of the ARCHER UK National Supercomputing Service (http://www.archer.ac.uk) through the Materials Chemistry Consortium via the EPSRC Grant No. EP/L000202 and the EPSRC-funded Centre for Doctoral Training in Advanced Characterisation of Materials for a studentship (EP/L015277/1).

\section{Author contributions}

C.G.S. designed the project; J.J.S. and P.H. conducted the lab-based experiments and performed data analyses; C.G.S., J.J.S, M.H. and C.L.B. carried out the neutron diffraction experiments; C.G.S. analysed the neutron diffraction data; S.T.B. and C.G.S developed the statistical mechanics aspects of this work; DFT calculations were carried out by B.S.; C.G.S, S.T.B., B.S. and J.J.S. wrote the manuscript and prepared the figures; all authors discussed the results and commented on the manuscript.

\section{Additional information}

Supplementary information is available in the online version of the paper. Correspondence and requests for materials should be addressed to C.G.S. (c.salzmann@ucl.ac.uk)

\section{Competing financial interests}


The authors declare no competing financial interests.

\section{Figures}
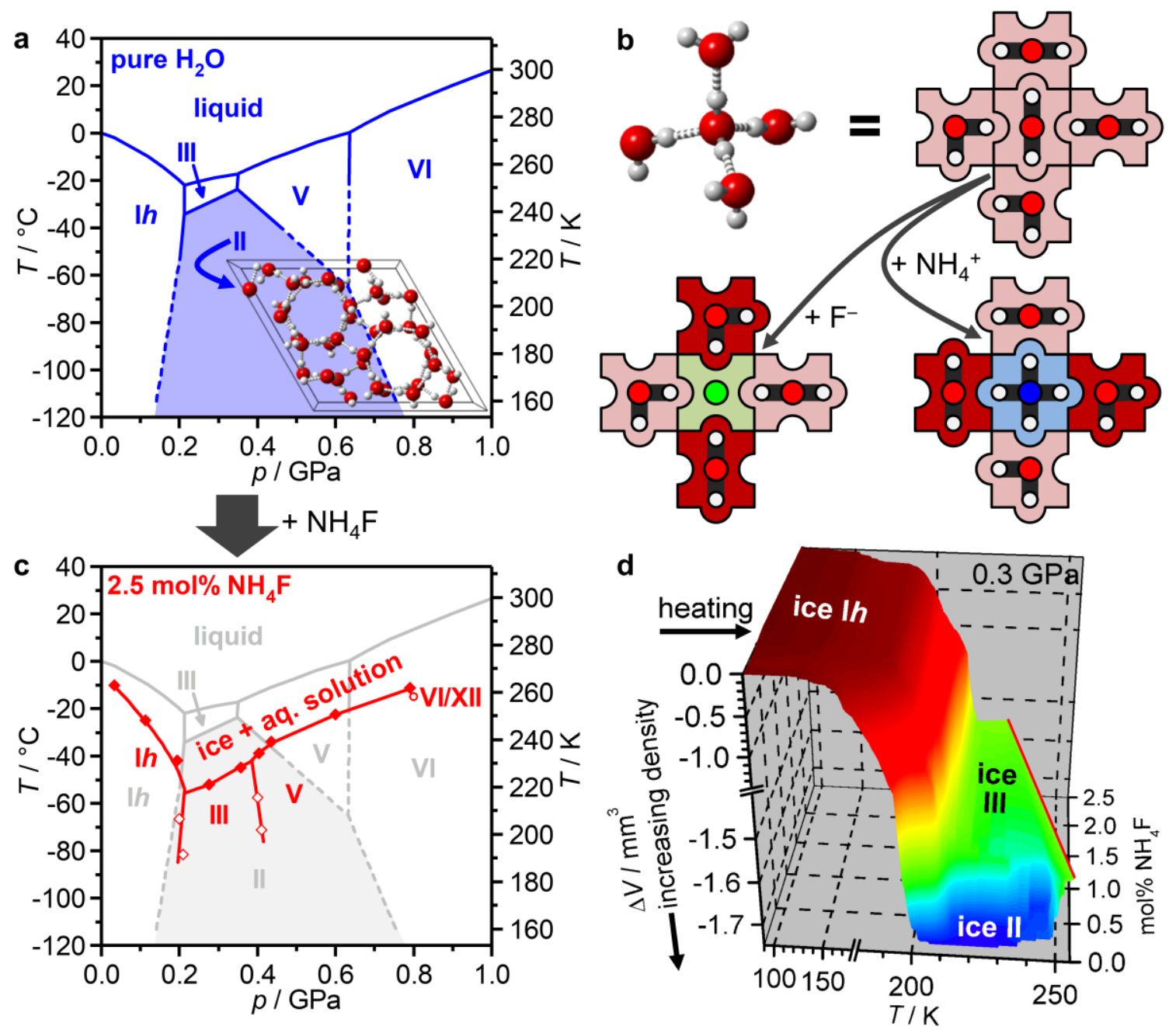

Figure 1. Tiny amounts of ammonium fluoride $\left(\mathrm{NH}_{4} \mathrm{~F}\right)$ cause ice II to disappear from water's phase diagram. (a) Temperature-pressure phase diagram of pure $\mathrm{H}_{2} \mathrm{O}$ with ice phases denoted by Roman numerals (adapted from ref. 6). The dashed phase boundaries are extrapolated. The crystal structure of the antiferroelectric hydrogen-ordered ice II with covalent $\mathrm{O}-\mathrm{H}$ bonds as solid lines and hydrogen bonds as dashed lines, respectively. ${ }^{29}$ (b) Schematic illustration of the reorientations of neighbouring water molecules (highlighted in dark red) in ice due to the replacement of $\mathrm{H}_{2} \mathrm{O}$ with $\mathrm{NH}_{4}{ }^{+}$or $\mathrm{F}^{-}$. Circles indicate atoms of oxygen (red), hydrogen (white), fluorine (green) and nitrogen (blue). (c) Phase diagram of $\mathrm{H}_{2} \mathrm{O}$ with $2.5 \mathrm{~mol} \% \mathrm{NH}_{4} \mathrm{~F}$ compared to that of pure $\mathrm{H}_{2} \mathrm{O}$ (background). The phase boundaries were determined using a piston-cylinder setup, and the phase identities were 
confirmed with X-ray diffraction as described in the Supplementary Information Section 1 . The onset temperatures of melting and solid-solid phase transitions are indicated by closed and open diamonds, respectively. (d) Nominal volume changes upon heating metastable ice $\mathrm{I} h / \mathrm{NH}_{4} \mathrm{~F}$ solid solutions at $0.3 \mathrm{GPa}$ in the 0 to $2.5 \mathrm{~mol}^{2} \mathrm{NH}_{4} \mathrm{~F}$ range. The red line indicates the onset of melting.
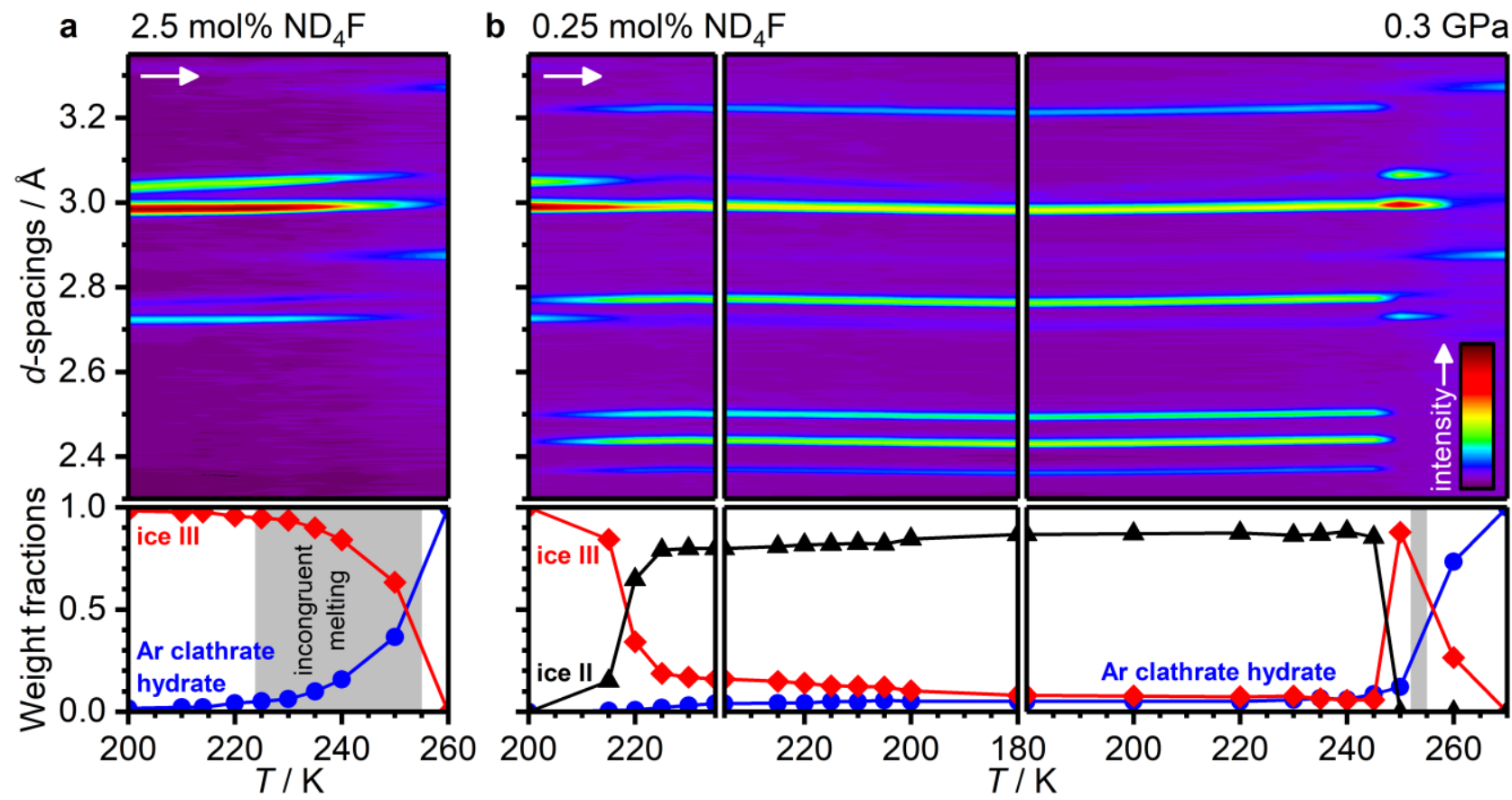

Figure 2. Phase transitions of $\mathrm{D}_{2} \mathrm{O}$ ice containing deuterated ammonium fluoride $\left(\mathrm{ND}_{4} \mathrm{~F}\right)$ at a pressure of $0.300 \mathrm{GPa}$. The powder neutron diffraction data was collected for ice with (a) $2.5 \mathrm{~mol} \%$ $\mathrm{ND}_{4} \mathrm{~F}$ and (b) $0.25 \mathrm{~mol} \% \mathrm{ND}_{4} \mathrm{~F}$. The arrows indicate the starting points of heating. The scale bar applies to all panels. The weight fractions of the crystalline components including ice III (red diamonds), ice II (black triangles) and CS-II argon clathrate hydrate (blue circles) at the given temperatures are shown in the lower part of the figure. The errors of the weight fractions (standard deviations) were estimated from the errors in the recorded diffraction intensities and found to be much smaller than the size of the symbols in (a) and (b). Error bars have therefore been omitted. The temperature ranges of melting are indicated by grey shaded areas. See Supplementary Information Sections 2 for full details on the analysis of the neutron diffraction data. 


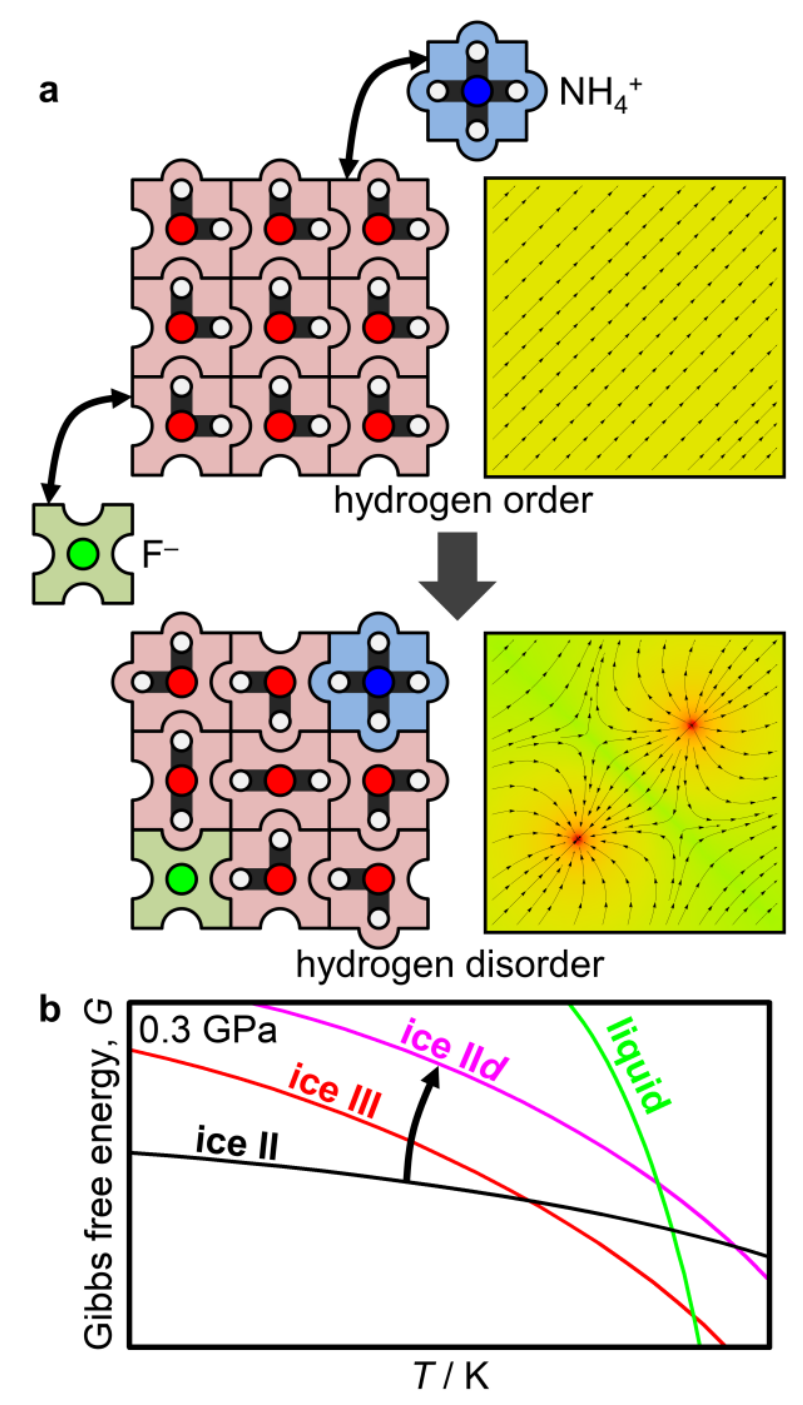

Figure 3. Topological and thermodynamic principles by which ice II becomes unstable in the presence of $\mathrm{NH}_{4} \mathrm{~F}$. (a) Ice II represented schematically as hydrogen-ordered 'square ice'. The orientational order of the water molecules is disrupted by substitution of $\mathrm{F}^{-}$and $\mathrm{NH}_{4}{ }^{+}$ions. Circles indicate atoms of oxygen (red), hydrogen (white), fluorine (green) and nitrogen (blue). The right panels 'zoom out' to show the conserved flux of the hydrogen-displacement field in the orientationally ordered state (top) and with two distant dopant ions (bottom). The dopants behave as topological defects that disrupt orientational order over long distances. (b) Free energy versus temperature curves for competing phases. The ice II curve shifts on doping towards the ice II $d$ curve (up arrow). 


\section{Methods}

Ammonium fluoride (99.99\% trace metal basis) and $\mathrm{D}_{2} \mathrm{O}(99.9 \% \mathrm{D})$ were purchased from Sigma Aldrich. Deuterated ammonium fluoride was prepared by dissolving $\mathrm{NH}_{4} \mathrm{~F}$ in an excess of $\mathrm{D}_{2} \mathrm{O}$ and completely evaporating the $\mathrm{D}_{2} \mathrm{O}$ under nitrogen three times. All ammonium fluoride solutions were prepared and stored in polyethylene containers. The ice samples were prepared by quickly pipetting the aqueous solutions into an indium cup inside a piston cylinder setup precooled with liquid nitrogen. This was followed by isobaric heating or compression experiments using a 30-tonne hydraulic press. Changes in sample volume were detected using a GT5000RA-L25 positional transducer from RDP electronics. After preparation of the high-pressure phases, some of the samples were quenched under pressure with liquid nitrogen and recovered at ambient pressure. Xray powder diffraction was performed using a custom-made sample holder with Kapton windows mounted on a Stoe Stadi-P transmission diffractometer with Cu radiation at $40 \mathrm{kV}, 30 \mathrm{~mA}$, monochromated by a Ge 111 crystal. Data were collected using a Mythen $1 \mathrm{~K}$ linear detector and the temperature of the sample was controlled with an Oxford Instruments CryojetHT. For in-situ pressure neutron diffraction measurements on the PEARL beamline ${ }^{30}$ at the ISIS facility, ground ice samples were transferred under liquid nitrogen into tubular TiZr cans which were pressure sealed and mounted inside a cryostat on the beamline. The pressure acting onto the samples was generated with a gas-compressor setup using argon gas as the pressure medium.

\section{Data availability}

The neutron diffraction data shown in Figures 2 and S3 can be accessed at https://data.isis.stfc.ac.uk/doi/investigation/64414858. All other data is available from the corresponding author upon request.

\section{References}


30 Bull, C. L. et al. PEARL: the high pressure neutron powder diffractometer at ISIS. High Pressure Res., 1-19, (2016). 